\section{Peso insuficiente ao nascer e crescimento alcançado na idade pré-escolar, por crianças atendidas em creches filantrópicas do município de Santo André, São Paulo, Brasil}

\author{
Insufficient weight at birth and preschool- \\ age growth in children attending charitably \\ funded crèches in the municipality of Santo \\ André, in the State of São Paulo, Brazil
}

Renato Minoru Yamamoto 1 Denise de Oliveira Schoeps 2 Luiz Carlos de Abreu 3 Claudio Leone 4
1 Centro de Saúde Escola "Prof. Samuel B. Pessoa". Universidade de São Paulo. São Paulo, SP, Brasil.

2 Departamento de Pediatria. Faculdade de Medicina do ABC. Santo André, SP, Brasil.

3,4 Departamento de Saúde Materno-Infantil. Faculdade de Saúde Pública. Universidade de São Paulo. Av. Dr. Arnaldo 715, sala 218 São Paulo, SP, Brasil. CEP: 01.246-904.

E-mail: leone.claudio@gmail.com

\begin{abstract}
Objectives: to compare rate of growth and prevalence of low stature at preschool age in children who were underweight at birth and in children born with a normal weight.

Methods: the study covered 323 children born underweight and 886 born with a normal weight from low-income families in fifteen charitably funded crèches in the municipality of Santo André, in the State of São Paulo, Brazil, in 2001 and 2002. The variables used were the $z$ score for weight and height for age, using the Center for Disease Control (CDC) and National Center for Health Statistics (NCHS) 2000 benchmark, comparing the means for the two groups. The prevalence ratio was calculated for low stature in preschool age children resulting from insufficient weight at birth.

Results: the z score for weight was -0.09 and 0.39 and the average $z$ score for height was -0.06 and 0.24 for underweight and normal weight at birth, respectively. The prevalence of low stature was $2.78 \%$ for children born underweight and $0.79 \%$ for normal weight. The PR for low stature at preschool age for children born underweight was 3.5 (CI95\% 1.3-9.4).

Conclusions: children born underweight had a lower rate of growth and greater risk of developing a growth disorder up to preschool age, suggesting a negative effect of insufficient birth weight on child growth.
\end{abstract}

Key words Growth, Birth weight, Child, preschool, Stature by age, Weight by age

\section{Resumo}

Objetivos: comparar o crescimento alcançado e a prevalência de baixa estatura na idade pré-escolar entre recém-nascidos de peso insuficiente (PNI) e de peso adequado (PNA).

Métodos: foram estudadas 323 crianças com PNI e 886 com PNA de famílias de baixa renda de quinze creches filantrópicas de Santo André, São Paulo, Brasil, em 2001 e 2002. As variáveis foram o escore $Z$ de peso e de estatura para a idade, pelo referencial Center for Disease Control (CDC) and National Center for Health Statistics (NCHS) 2000, comparando-se as médias dos dois grupos. Calculou-se a razão de prevalência $(R P)$ de baixa estatura na idade préescolar em decorrência do peso insuficiente ao nascer.

Resultados: o escore z médio de peso foi $-0,09$ e 0,39 e o escore $z$ médio de estatura foi de -0,06 e de 0,24 para as crianças de PNI e de PNA, respectivamente. A prevalência de baixa estatura foi de 2,78\% para as crianças com PNI e de 0,79\% para aquelas com PNA. A RP de baixa estatura na idade préescolar para as crianças de PNI foi de 3,5 (IC95\% 1,3-9,4).

Conclusões: as crianças com PNI apresentaram crescimento inferior e maior risco de falhas de crescimento até a idade pré-escolar, sugerindo um efeito negativo do peso insuficiente ao nascer sobre o crescimento infantil.

Palavras-chave Crescimento, Peso ao nascer, Pré-escolar, Estatura-idade, Peso-idade 


\section{Introdução}

As crianças com baixo peso ao nascer (BPN) frequentemente são alvo de seguimento e cuidados diferenciados, devido a problemas como a elevada morbimortalidade e ao maior risco de atraso de crescimento e desenvolvimento. ${ }^{1-3}$ Entretanto, pouca atenção tem sido dada às crianças nascidas com peso insuficiente (PNI), cujo peso de nascimento é igual ou maior do que $2500 \mathrm{~g}$ e menor do que $3000 \mathrm{~g}$, que, de forma igual, teriam potencialmente maiores chances de virem a ser alvo de uma assistência diferenciada. Apesar dos recém-nascidos com PNI apresentarem menor morbimortalidade quando comparados aos nascidos com BPN, a sua incidência é alta em países em desenvolvimento. No Brasil, os nascimentos de recém-nascidos com PNI representam cerca de $1 / 3$ do total de nascimentos, sendo três a quatro vezes mais elevada a incidência do que os nascimentos de crianças com BPN.4-5

Os recém-nascidos com PNI, assim como os de BPN, constituem um grupo heterogêneo, que inclui desde prematuros com peso adequado ao nascer até recém-nascidos a termo com restrição de crescimento intrauterino (RCIU). Em populações com baixo nível socioeconômico, a RCIU assume grande importância epidemiológica, podendo representar até 80\% dos casos de PNI.6-7 Nessas populações, a má nutrição materna é um dos fatores associados à RCIU mais importantes.7-8 Nos países desenvolvidos, a RCIU usualmente representa cerca de $50 \%$ dos casos de PNI, sendo a insuficiência placentária a principal causa.6-7

Estudos realizados em crianças com BPN mostram que a velocidade de crescimento em peso e estatura no primeiro ano de vida, particularmente no primeiro semestre, é maior do que a observada nas crianças nascidas com peso adequado (PNA). Porém, apesar do crescimento compensatório ("catch-up growth") observado nessa população, as prevalências de baixa estatura e de baixo peso nessas crianças, em idades maiores, são frequentemente mais elevadas, inclusive na adolescência.9-14

Apesar de a maioria dos estudos investigarem o crescimento e a evolução nutricional de recémnascidos com BPN e pequenos para a idade gestacional,15-19 ainda são escassos os estudos avaliando o crescimento e a evolução do estado nutricional de recém-nascidos das crianças com PNI.

Pesquisa realizada no município de São Paulo, que avaliou o crescimento de crianças com PNI, ${ }^{20}$ observou que a evolução ponderal e de crescimento linear foi insuficiente até os dois anos de idade, tendo-se encontrado valores médios de peso e comprimento nesta idade inferiores à mediana do referencial do National Center for Health Statistics (NCHS), de 1978.21 Ao analisarem a influência das condições de vida, os autores constataram que as crianças com PNI submetidas a piores condições de vida apresentavam evolução ponderal e crescimento linear ainda mais comprometidos, se comparados ao grupo de PNI como um todo.

As creches são hoje uma realidade na vida de uma grande parcela das crianças brasileiras em idade pré-escolar, onde permanecem cerca de oito horas por dia, cinco dias por semana. Entre as principais características de atuação das creches no Brasil, destacam-se o desenvolvimento de atividades educativas, assistenciais e de promoção de saúde. 22 Dessa forma, desde que a frequência da criança seja adequada, esses locais que podem propiciar boas condições para garantir o desenvolvimento do potencial de crescimento e para manter um adequado estado nutricional, até o início da idade escolar.23,24

Entre os dois e os sete anos de idade, a velocidade de crescimento é menor do que a observada nos primeiros dois anos de vida, observando-se, em média, para indivíduos que não estejam em processo de crescimento compensatório, um incremento médio anual de cinco centímetros na altura e de dois quilos, no peso. $25 \mathrm{~A}$ partir da idade de dois anos, não há estudos no Brasil avaliando o crescimento alcançado e o estado nutricional de crianças nascidas com PNI que possibilitem verificar se a retomada de crescimento ocorrida de forma incompleta até os dois anos de idade, continua a ocorrer até o início da idade escolar. Desse modo, torna-se importante comparar o crescimento alcançado pelas crianças nascidas com PNI, entre os dois e seis anos de idade, em relação às crianças com PNA.

\section{Métodos}

O estudo foi realizado nas quinze creches filantrópicas existentes em Santo André, município localizado na Região Metropolitana de São Paulo. Todas as creches são filiadas à Federação de Entidades Assistenciais de Santo André (FEASA) e apresentam cobertura assistencial da Fundação de Assistência à Infância de Santo André (FAISA), caracterizada pela atuação sistemática de médicos pediatras. Desde 1997, a FAISA também supervisiona o trabalho de agentes de saúde, que realizam ações de promoção de saúde nestas instituições, inclusive aquelas voltadas para a vigilância do crescimento e do estado nutricional. O período de estudo correspondeu aos anos de 2001 e 2002. No caso das crianças que frequentaram a creche nos dois 
anos, foram utilizados apenas os dados antropométricos obtidos no ano de 2002. Efetuou-se o levantamento retrospectivo de dados a partir da análise dos prontuários, nos quais estão registradas todas as informações relativas às avaliações realizadas pelos profissionais responsáveis pelas ações de saúde em cada creche. As informações sobre o peso ao nascer foram obtidas a partir do cartão do recém-nascido fornecido pela maternidade $\mathrm{e}$ registradas no prontuário da criança na ocasião da admissão à creche. Os dados relativos à idade materna, à renda familiar per capita, ao número de pessoas residentes na casa e a duração do aleitamento materno foram obtidos durante a matrícula da criança. Todos os registros foram efetuados por profissionais treinados. Somente crianças nascidas a termo foram incluídas no estudo. Foram excluídas as crianças sem registro de peso de nascimento no prontuário e as portadoras de doenças crônicas reconhecidamente limitadoras do crescimento, tais como: doenças cromossômicas, cardiopatias com repercussão hemodinâmica, doenças endócrinas graves (síndrome de Cushing, panhipopituitarismo, deficiência de hormônio do crescimento, puberdade precoce), mucoviscidose, neoplasias, etc. Após o censo, as crianças foram classificadas de acordo com o peso ao nascer, compondo dois grupos de estudo: o universo de 323 crianças nascidas com peso insuficiente, entre 2500 e 3000 g (PNI), e o universo de 886 crianças nascidas com peso entre 3000 e $3800 \mathrm{~g}$ (PNA).

As medidas antropométricas (peso e estatura) foram tomadas por profissionais treinados especificamente, sob supervisão da equipe de pediatras durante a rotina e segundo técnicas internacionalmente preconizadas. $20 \mathrm{~A}$ aferição do peso foi realizada em balança mecânica tipo pesa-bebê, com capacidade de 15 quilos e subdivisões de 10 gramas. As crianças com peso $>15$ quilos foram pesadas em balança mecânica tipo plataforma, com capacidade de 150 quilos e subdivisões de 100 gramas, ambas de marca Filizola ${ }^{\circledR}$. O comprimento foi medido com antropômetro de madeira, no caso de crianças menores de $95 \mathrm{~cm}$, com escala de fita métrica metálica. As crianças com estatura $>95 \mathrm{~cm}$ foram medidas em antropômetro feito em haste não flexível de alumínio fixo à parede, a prumo, cuja escala era uma fita métrica de fibra de vidro inextensível. Ambos os instrumentos têm divisão em centímetros e milímetros. O registro das medidas antropométricas foi feito em formulários padronizados para o atendimento nas unidades, na sala de antropometria. Considerou-se a primeira medida observada no período correspondente ao estudo. As crianças que ingressaram nas creches em 2001 e 2002 tiveram dados antropométricos coletados nos dois primeiros meses de sua frequência à creche.

As variáveis de estudo foram o peso para a idade $(\mathrm{P} / \mathrm{I})$ e a altura para a idade (A/I). ${ }^{20}$ No caso das crianças há mais tempo na creche, as medidas foram obtidas no início de 2001.

Considerando o número de crianças em cada grupo (PNI e PNA), houve verificação se o número seria suficiente para detectar uma diferença de pelo menos um quinto de desvio-padrão entre as médias de P/I e A/I, com um poder de teste de $90 \%$ e um valor de alfa. $=5 \%$, Esta estimativa foi realizada utilizando o software Statmate da Graphpad.

Os dados relativos ao peso, estatura, sexo e idade foram transportados para um banco de dados criado no software Acess e analisados utilizando o programa Excel. O cálculo dos escores $\mathrm{Z}$ de peso e de estatura por sexo e idade foi realizado utilizando o programạ Epi Info 2000. Foram calculadas as médias e desvios-padrão das variáveis antropométricas e a distribuição das variáveis categóricas. Para comparação das médias de entre nos grupos com PNI e PNA por idade, em cada idade, foram utilizados o teste $\mathrm{t}$ de Student e, para dados não paramétricos o de Mann-Whitney. Foram considerados estatisticamente significantes valores de $p<0,05.26$

A baixa estatura e peso baixo foram respectivamente definidos como escore $\mathrm{Z}$ de estatura para a idade ou de peso para a idade inferiores a -2 (Organização Mundial da Saúde - OMS).20

Foi calculada a razão de prevalência (RP) de baixa estatura e de baixo peso na idade pré-escolar entre os dois grupos de peso de nascimento: insuficiente e adequado.

Todas as análises estatísticas foram realizadas no software SPSS 12.0.

Este estudo foi aprovado pela Comissão de Ética para Análise de Projetos de Pesquisa do Hospital das Clínicas da Faculdade de Medicina da Universidade de São Paulo em 2004, Protocolo CAPPesq $n^{\circ}$ 0923/04.

\section{Resultados}

Do total de 1544 crianças matriculadas, 335 (21,7\%) foram excluídas pelos seguintes motivos: quatro crianças $(0,3 \%)$ por apresentarem menos de dois anos de idade, sete $(0,5 \%)$ por falta de informação de idade gestacional, 39 (2,5\%) por falta de informação quanto ao peso de nascimento, $45(2,9 \%)$ por prematuridade (associada ou não ao baixo peso ao 
nascer), $94(6,1 \%)$ por baixo peso ao nascer (sem prematuridade) e $147(9,5 \%)$ por apresentarem peso de nascimento superior a 3800 gramas. Restaram assim 1209 crianças que preenchiam os critérios para serem incluídas no estudo; destas 323 (26,7\%) haviam nascido com PNI e $886(73,3 \%)$ com PNA.

A média de peso ao nascer no grupo com PNI foi $-1,3$ escore $Z( \pm 0,2)$, enquanto o peso médio ao nascer no grupo com PNA foi $-0,1$ escore $Z( \pm 0,5)$.

Entre as crianças com PNI, 173 eram do sexo masculino (53,6\%) e 150 do sexo feminino (46,4\%). No grupo com PNA, 494 eram meninos $(55,8 \%)$ e 392 eram meninas (44,2\%). Não houve diferença estatisticamente significante na distribuição do sexo entre os dois grupos. Todas as crianças eram provenientes de famílias de baixo nível socioeconômico, com renda familiar mensal inferior a um salário mínimo per capita.

A Tabela 1 mostra as características dos grupos de crianças com PNI e PNA, segundo o peso ao nascer, tempo total de aleitamento materno, tempo total de frequência à creche desde o nascimento, internações, idade materna ao nascimento da criança, renda familiar per capita e número de pessoas na casa. Exceto quanto ao peso ao nascer, os grupos são comparáveis quanto à distribuição das demais características individuais e familiares descritas.

A distribuição de PNI segundo a idade, nos grupos de estudo e comparação, encontra-se na Tabela 2. Em ambos os grupos havia proporcionalmente menos crianças entre os dois e os três anos de idade e mais crianças entre os cinco e os seis anos de idade.

A comparação dos valores médios de $\mathrm{P} / \mathrm{I}$ observados ao nascimento e na idade pré-escolar para as crianças com PNI mostra que houve um intenso crescimento ponderal compensatório. Ao nascimento, a média de $\mathrm{P} / \mathrm{I}$ foi igual a $-1,3$ e na idade préescolar foi de $0,09(p<0,001)$.

$\mathrm{O}$ crescimento alcançado pelas crianças com PNI na idade pré-escolar foi inferior ao observado para as crianças com PNA, tanto em P/I quanto em A/I. (Tabela 3)

A comparação realizada entre as crianças com PNI e PNA, segundo a subdivisão em grupos etários, mostrou diferenças estatisticamente significantes para A/I nas faixas etárias de três anos e para o peso e a estatura nos grupos de quatro e cinco anos de idade. (Tabela 4)

No grupo de crianças com PNI, nove tinham baixa estatura, o que correspondeu a uma prevalência igual a $2,78 \%$. Entre as crianças com PNA, sete eram de baixa estatura, com uma prevalência de $0,79 \%, 1 / 3$ da observada para as crianças com PNI.

A prevalência de baixa estatura para idade foi 3,5 (IC95\%: 1,3-9,4) vezes mais elevada nas crianças com PNI do que as nascidas com PNA, resultado estatisticamente significante $(p<0,016)$. Com relação ao peso, embora se tenha constatado associação positiva entre PNI e baixo peso ( $\mathrm{RP}=2,1$; IC95\%: $0,8-5,7)$, a associação não foi significante.

Tabela 1

Médias e desvio-padrão $( \pm D P)$ das características da população de estudo segundo peso ao nascer (PNI e PNA). Município de Santo André, SP, 2001-2002.

\begin{tabular}{|c|c|c|c|}
\hline \multirow[t]{3}{*}{ Características } & \multicolumn{2}{|c|}{ GRUPO } & \multirow{3}{*}{$p^{*}$} \\
\hline & PNI & PNA & \\
\hline & $\bar{X} \pm D P$ & $\bar{X} \pm D P$ & \\
\hline Aleitamento materno (tempo total em meses) & $8,2 \pm 10,0$ & $8,7 \pm 9,9$ & 0,438 \\
\hline Tempo de creche (meses) & $17,9 \pm 16,0$ & $18,0 \pm 16,9$ & 0,926 \\
\hline Internações (número) & $0,4 \pm 0,9$ & $0,3 \pm 0,9$ & 0,087 \\
\hline Idade materna (anos) & $25,6 \pm 6,7$ & $25,6 \pm 6,5$ & 0,999 \\
\hline Renda familiar per capita (reais) & $116,2 \pm 71,5$ & $112,9 \pm 75,8$ & 0,496 \\
\hline Número de pessoas na casa & $4,6 \pm 1,7$ & $4,6 \pm 1,6$ & 0,999 \\
\hline
\end{tabular}

PNI = Peso ao nascer insuficiente; PNA = Peso ao nascer adequado; *Teste U de Mann-Whitney. 
Distribuição das crianças do PNI e PNA, segundo a idade e o sexo.

\begin{tabular}{|c|c|c|c|c|c|c|c|c|}
\hline \multirow[t]{3}{*}{ Características } & \multicolumn{4}{|c|}{ Grupos } & \multicolumn{2}{|c|}{ Total $(\mathrm{N}=1209)$} & \multirow[t]{3}{*}{$\chi^{2}$} & \multirow[t]{3}{*}{$p$} \\
\hline & \multicolumn{2}{|c|}{ PNI (N=323) } & \multicolumn{2}{|c|}{ PNA $(\mathrm{N}=886)$} & \multirow[b]{2}{*}{$\mathrm{n}$} & \multirow[b]{2}{*}{$\%$} & & \\
\hline & $\mathrm{n}$ & $\%$ & $\mathrm{n}$ & $\%$ & & & & \\
\hline \multicolumn{9}{|l|}{ Idade (anos) } \\
\hline $2-3$ & 25 & 7,8 & 84 & 9,5 & 109 & 9,0 & 0,675 & 0,411 \\
\hline $3-4$ & 69 & 21,3 & 166 & 18,7 & 235 & 19,4 & 0,882 & 0,347 \\
\hline $4-5$ & 67 & 20,7 & 225 & 25,4 & 292 & 24,1 & 2,548 & 0,110 \\
\hline $5-6$ & 91 & 28,2 & 251 & 28,3 & 342 & 28,3 & 0,003 & 0,957 \\
\hline $6-7$ & 71 & 22,0 & 160 & 18,1 & 231 & 19,1 & 2,110 & 0,146 \\
\hline \multicolumn{9}{|l|}{ Sexo } \\
\hline Masculino & 173 & 53,6 & 494 & 55,8 & 667 & 55,2 & 0,377 & 0,539 \\
\hline Feminino & 150 & 46,4 & 392 & 44,2 & 542 & 44,8 & & \\
\hline
\end{tabular}

PNI = Peso ao nascer insuficiente; PNA = Peso ao nascer adequado.

Tabela 3

Médias e desvios-padrão ( $\pm D P$ ) das variáveis antropométricas (escores $Z$ de altura para idade e peso para idade) no grupo total de PNI e de PNA, alcançadas na idade pré-escolar.

Variáveis antropométricas (escore Z)

Grupos

\begin{tabular}{|c|c|c|c|c|}
\hline \multicolumn{2}{|c|}{ PNI $(\mathrm{N}=323)$} & \multicolumn{2}{|c|}{ PNA $(\mathrm{N}=886)$} & \multirow[t]{2}{*}{$p^{*}$} \\
\hline $\bar{x} \pm D P$ & Md & $\bar{x} \pm D P$ & $\mathrm{Md}$ & \\
\hline$-0,06 \pm 1,06$ & $-0,09$ & $0,24 \pm 0,97$ & 0,23 & $<0,001$ \\
\hline $0,09 \pm 1,06$ & 0,09 & $0,39 \pm 1,00$ & 0,39 & $<0,001$ \\
\hline
\end{tabular}

$\mathrm{PNI}=$ Peso ao nascer insuficiente; PNA = Peso ao nascer adequado; ${ }^{*}$ teste $\mathrm{t}$ de Student não pareado; Md=mediana. 
Tabela 4

Distribuição das médias e desvios-padrão ( $(\mathrm{DP})$ das variáveis antropométricas, nos PNI e PNA, segundo a idade.

\begin{tabular}{|c|c|c|c|c|c|c|c|c|c|c|c|c|}
\hline \multirow[t]{3}{*}{ Idade (anos) } & \multicolumn{7}{|c|}{ PNI } & \multicolumn{5}{|c|}{ PNA } \\
\hline & \multicolumn{3}{|c|}{ Escore Z A/I } & \multicolumn{3}{|c|}{ Escore Z P/I } & \multicolumn{3}{|c|}{ Escore Z A/I } & \multicolumn{3}{|c|}{ Escore Z P/I } \\
\hline & $\mathrm{n}$ & $\bar{x} \pm D P$ & Md & $\mathrm{n}$ & $\bar{x} \pm D P$ & Md & $\mathrm{n}$ & $\bar{X} \pm D P$ & Md & $\mathrm{n}$ & $\bar{x} \pm D P$ & $\mathrm{Md}$ \\
\hline 2 a 2,9 & 25 & $-0,30 \pm 0,87$ & $-0,33$ & 25 & $-0,16 \pm 1,28$ & $-0,14$ & 84 & $-0,03 \pm 0,88$ & $-0,10$ & 84 & $0,15 \pm 0,93$ & 0,11 \\
\hline 3 a 3,9 & 69 & $-0,10 * \pm 1,09$ & $-0,20$ & 69 & $0,08 \pm 1,11$ & $-0,02$ & 166 & $0,22 \pm 1,00$ & 0,19 & 166 & $0,34 \pm 1,08$ & 0,25 \\
\hline 4 a 4,9 & 67 & $-0,03^{*} \pm 1,02$ & $-0,17$ & 67 & $0,03^{*} \pm 0,90$ & $-0,01$ & 225 & $0,33 \pm 1,00$ & 0,30 & 225 & $0,44 \pm 0,98$ & 0,41 \\
\hline 5 a 5,9 & 91 & $-0,07 * \pm 1,00$ & $<0,001$ & 91 & $0,04^{*} \pm 1,03$ & 0,08 & 251 & $0,23 \pm 0,94$ & 0,24 & 251 & $0,40 \pm 0,99$ & 0,50 \\
\hline 6 a 6,9 & 71 & $0,07 \pm 1,18$ & $<0,001$ & 71 & $0,28 \pm 1,08$ & 0,31 & 160 & $0,27 \pm 0,98$ & 0,29 & 160 & $0,49 \pm 0,99$ & 0,59 \\
\hline
\end{tabular}

PNI=Peso ao nascer insuficiente; PNA=Peso ao nascer adequado; * $p<0,05$ (comparações entre PNI e PNA) -teste $t$ de Student; Md=mediana.

Tabela 5

Prevalência de baixa estatura e baixo peso em crianças pré-escolares, segundo o seu peso de nascimento.

\begin{tabular}{|c|c|c|c|c|c|c|c|c|c|c|}
\hline & \multicolumn{5}{|c|}{ Estatura para a idade } & \multicolumn{5}{|c|}{ Peso para a idade } \\
\hline & \multicolumn{2}{|c|}{ Baixa estatura* } & \multicolumn{2}{|c|}{ Estatura normal } & \multirow[t]{2}{*}{$\mathrm{RP} *(\mathrm{IC} 95 \%)$} & \multicolumn{2}{|c|}{ Baixo peso** } & \multicolumn{2}{|c|}{ Sem Peso Baixo } & \multirow[t]{2}{*}{$\mathrm{RP} * *(\mathrm{IC} 95 \%)$} \\
\hline & $\mathrm{n}$ & $\%$ & $\mathrm{n}$ & $\%$ & & $\mathrm{n}$ & $\%$ & $n$ & $\%$ & \\
\hline PN Insuficiente & 9 & 2,78 & 314 & 97,22 & $3,5(1,3-9,4)$ & 7 & 2,16 & 316 & 97,84 & $2,1(0,8-5,7)$ \\
\hline PN Adequado & 7 & 0,79 & 879 & 99,12 & & 9 & 1,01 & 877 & 98,99 & \\
\hline Total & 16 & 1,34 & 1193 & 98,76 & & 16 & 1,34 & 1193 & 98,76 & \\
\hline
\end{tabular}

*Teste exato de Fischer $p<0,016 ; * *$ Teste exato de Fischer $p<0,152 ; \mathrm{RP}=$ Razão de Prevalência; PN=Peso ao nascer.

\section{Discussão}

No presente estudo, todas as crianças do grupo com PNI eram nascidas a termo, o que permitia pressupor que o nascimento com peso insuficiente era decorrente de uma restrição de crescimento intrauterino. Esse fato corrobora, em parte, pelo crescimento compensatório ocorrido após o nascimento, deixando menos plausível a herança genética como determinante do crescimento desfavorável. Os resultados mostraram que as crianças com PNI apresentaram um crescimento compensatório entre o nascimento e a faixa etária pré-escolar, pois a classificação de crescimento alcançado na idade pré-escolar foi melhor do que a verificada ao nascimento dessas crianças. O fenômeno conhecido como crescimento compensatório é observado após períodos da vida intrauterina, infância ou adolescência caracterizados por agravos à saúde, de origem infecciosa, por exemplo, durante os quais não ocorreu o crescimento normal esperado para a idade, sexo e herança genética. No período de crescimento compensatório, a criança apresenta ganho de peso e de estatura superior ao esperado para crianças normais de mesma idade e sexo. Para que esse fenômeno ocorra, é necessário que o aporte de calorias, proteínas e de minerais, particularmente o ferro, seja maior do que o recomendado para a respectiva faixa etária. ${ }^{27}$ Também é fundamental que os fatores protetores do crescimento estejam atuando na vida da criança, inclusive os fatores de proteção alimentar, anti- 
infecciosa, do ambiente físico, da casa ou da creche, e particularmente a proteção afetiva conferida pelos pais, irmãos, demais membros familiares e cuidadores da creche.

Ao se comparar o crescimento das crianças com PNI com a distribuição dos valores de peso e de estatura do referencial utilizado (CDC/NCHS), seria possível supor que as mesmas tiveram um crescimento pôndero-estatural adequado, pois esses valores praticamente se sobrepõem aos do referencial. Na análise da distribuição do peso para a idade, os valores estão deslocados para a direita, indicando inclusive uma distribuição ligeiramente superior a do $\mathrm{CDC} / \mathrm{NCHS}$. Entretanto, ser um referencial recomendado para uso universal não é sinônimo de padrão de normalidade de crescimento. Como ideal, para as populações com padrões étnicos distintos ou de diferentes condições de vida, o melhor referencial de crescimento é aquele obtido a partir de valores observados para os seus pares, para determinar se o crescimento atingido, em condições socioeconômicas e ambientais semelhantes, é o melhor possível.

No presente estudo, abstraindo do referencial, a comparação do crescimento das crianças de PNI com as crianças de PNA permite observar que o crescimento das primeiras fica aquém do atingido pelas crianças com PNA, tanto em peso quanto em estatura. Tanto as crianças com PNI quanto as com PNA apresentavam histórias de vida semelhantes. Foram amamentadas em média até os oito meses de idade, praticamente não necessitaram de internações, eram provenientes de famílias com características socioeconômicas e culturais semelhantes, com baixa renda e, em média, quatro pessoas, cujas mães possuíam em média 25 anos de idade por ocasião do nascimento das crianças. As crianças com PNI e com PNA receberam os mesmos cuidados na creche que frequentavam, onde estavam matriculadas por períodos de tempo semelhantes. Em ambos os grupos, foram também excluídas todas as crianças com doenças que comprometessem o crescimento. Apesar de todas essas características serem semelhantes, as crianças com PNI alcançaram crescimento inferior às crianças com PNA, até a faixa etária dos seis anos de idade.

Por se tratar de grupos procedentes de condições socioeconômicas muito semelhantes, pode-se supor que as diferenças observadas no crescimento de crianças com PNI e com PNA sejam, pelo menos em parte, relacionadas ao peso de nascimento e, portanto, mais especificamente ao crescimento inadequado ocorrido na vida intrauterina.

Estudo descritivo analisou o crescimento pós- natal de gêmeos discordantes monozigóticos, em que o peso de um dos gêmeos era pelo menos $30 \%$ maior do que o do outro, mostrando que, ao final da idade pré-escolar, ainda persistia uma diferença de peso e estatura entre eles de meio desvio-padrão ou mais. ${ }^{28,29}$ Nesses casos, a herança genética, o ambiente de vida e todos os cuidados prestados às crianças foram os mesmos e a condição denominada restrição de crescimento intrauterino, que diferenciava os pares de irmãos, teria sido a determinante das diferentes situações de crescimento na infância.

Os referenciais de crescimento utilizados em todo o mundo, que contêm curvas de peso, estatura e índice de massa corpórea, foram construídos a partir de estudos populacionais, que utilizaram medidas de indivíduos predominantemente com peso adequado ao nascer, estabelecendo os chamados canais do crescimento normal, dentro dos quais as medidas individuais deveriam se manter a partir do nascimento. Dessa forma, esses referenciais acabam privilegiando a monitorização do crescimento dos indivíduos de menor risco para falhas de crescimento, ou seja, aqueles nascidos com peso adequado. Obviamente, isso apresenta uma maior dificuldade para a monitorização do crescimento das crianças com peso de nascimento abaixo de $3000 \mathrm{~g}$, grupo que inclui as nascidas com peso insuficiente.

As crianças com baixo peso ao nascer ou nascidas pequenas para a idade gestacional recebem cuidados diferenciados nos serviços de neonatologia, em função de seu risco para distúrbios metabólicos nos primeiros dias de vida, e são acompanhadas em serviços ambulatoriais de Pediatria como crianças de maior risco de morbimortalidade no primeiro ano de vida. A partir do momento que superem o percentil correspondente ao ponto de corte adotado para a definição de desnutrição ou de baixa estatura, passam a ser consideradas como de crescimento normal. 30

As crianças com PNI, que não possuem as formas mais graves de RCIU, são habitualmente classificadas como crianças sem risco para desnutrição ou para falhas de crescimento no processo de monitorização de crescimento realizado em unidades ambulatoriais de Pediatria. Como possuem ao nascimento - devido à RCIU leve, mas de longa duração - comprometimento proporcional de peso e estatura, acabam sendo consideradas constitucionalmente de menor potencial de crescimento e as orientações realizadas junto à mãe ou responsável pelos cuidados da criança são direcionadas apenas para manter, ou não piorar, o canal de crescimento que apresentam.

No seguimento das crianças com BPN ou com PNI, para que o diagnóstico de crescimento seja 
corretamente feito, é preciso considerar como parte da história natural do crescimento pós-natal desses indivíduos o chamado crescimento compensatório. Para preservar o potencial biológico de crescimento dessas crianças, deve ser objetivo das ações de prevenção e promoção de saúde uma velocidade de ganho de peso e estatura um pouco superior à esperada, com uma tendência de crescimento que leve esses parâmetros antropométricos a se aproximarem da mediana do referencial de crescimento, no decorrer dos primeiros dois anos de vida. Assim, as crianças de PNI, que ao nascimento têm o peso e a estatura mais próximos ao percentil 10 do referencial de crescimento, deverão, nos primeiros anos de vida, ter uma tendência ascendente dos valores de peso e estatura em direção à mediana, ou seja, apresentando nesse período curvas pôndero-estaturais de inclinação maiores do que a curva correspondente à

\section{Referências}

1. Gomes JO, Santo AH. Infant mortality in a midwestern municipality of the State of Sao Paulo, Brazil, 1990 to 1992. Rev Saúde Pública. 1997; 31: 330-41.

2. Grantham-McGregor SM, Lira PI, Ashworth A, Morris SS, Assuncao AM. The development of low birth weight term infants and the effects of the environment in northeast Brazil. J Pediatr. 1998; 132: 661-6.

3. Souza RK, Gotlieb SL. Probability of dying in the first year of life in an urban area of southern Brazil. Rev Saúde Pública. 1993; 27: 445-54.

4. Almeida LAA, Barbieri MA, Gomes UA, Reis PM, Chiaratii TM, Vasconcelos V, Bettiol H. Peso ao nascer, classe social e mortalidade infantil em Ribeirão Preto, São Paulo. Cad Saúde Pública. 1992; 8: 190-8.

5. Leone C, Mascaretti LAS, Primo E, Yamamoto TS. Peso de nascimento e características médico-sociais. J Pediatr (Rio J). 1992; 68: 376-9.

6. Barros FC, Hutly SRA, Victora CG, Kirkwood BR, Vaughan JP. Comparison of the causes and consequences of prematurity and intrauterine growth retardation: a longitudinal study in southern Brazil. Pediatrics. 1992; 90:238-44.

7. Kramer MS. Determinants of low birth weight: methodological assessment and meta-analysis. Bull WHO. 1987; 65: 663-737.

8. Perez-Escamilla R, Pollitt E. Causes and consequences of intrauterine growth retardation in Latin America. Bull Pan Am Health Organ. 1992; 26: 128-47.

9. Ashworth A, Morris SS, Lira PI. Postnatal growth patterns of full-term low birth weight infants in Northeast Brazil are related to socioeconomic status. J Nutr. 1997; 127: 1950-6.

10. Chaussian JL, Colle M, Ducret JP. Adult height in children with prepubertal short stature secondary to intrauterine growth retardation. Acta Paediatr. (Suppl.) 1994; 399: 72-3. do percentil de origem da criança.

$\mathrm{O}$ crescimento alcançado pelas crianças com peso de nascimento insuficiente aqui avaliado foi inferior ao observado para as crianças com peso de nascimento adequado, tanto em peso quanto em estatura, até os seis anos de idade. A prevalência de baixa estatura na idade pré-escolar foi pequena na população estudada, entretanto, foi três vezes maior entre as crianças com peso de nascimento insuficiente. Caso novos estudos confirmem que, quando comparados a crianças de condição socioeconômica semelhante, mas que apresentaram PNA adequado, as crianças com PNI são de risco para falhas de crescimento até a idade pré-escolar, o PNI poderá se tornar um indicador de que essas crianças necessitam receber uma atenção diferenciada nos programas de vigilância do crescimento.

11. Karlberg J, Albertsson W. Growth in full-term small-forgestational-age infants: from birth to final height. Pediatr Res. 1995; 38: 733-9.

12. Karlberg J, Luo ZC. Foetal size to final height. Acta Paediatr. 2000; 89: 632-6.

13. Paz I, Seidman DS, Danon YL, Laor A, Stevenson DK, Gale R. Are children born small for gestational age at increased risk of short stature? Am J Dis Child. 1993; 147: 337-9.

14. Tuvemo T, Cnattingius S, Jonsson B. Prediction of male adult stature using anthropometric data at birth: a nationwide population-based study. Pediatr Res. 1999; 46: 491-5.

15. Albertsson-Wikland K, Karlberg J. Natural growth in children born small for gestational age with and without catchup growth. Acta Paediatr Suppl. 1994; 399: 64-70;

16. Fewtrell MS, Morley R, Abbott RA, Singhal A, Stephenson T, MacFadyen UM, Clements H, Lucas A. Catch-up growth in small-for-gestational-age term infants: a randomized trial. Am J Clin Nutr. 2001; 74: 516-23.

17. Hokken-Koelega, AC De Ridder MAJ, Lemmen H, Den Hartog H, De Muinck Keizer-Schrama SMPF, Drop SLS. Children born small for gestational age: do they catch up? Pediatr Res. 1995; 38: 267-71.

18. Tenovuo A, Kero P, Piekkala P, Korvenranta H, Silanpãã M, Erkkola R. Growth of 519 small for gestational age infants during the first two years of life . Acta Paediatr Scand. 1987; 76: 636-46.

19. Victora CG, Barros FC, Horta BL, Martorell R. Short-term benefits of catch-up growth for small-for-gestational-age infants. Int J Epidemiol. 2001; 30: 1325-30.

20. Yamamoto RM. Avaliação do crescimento de lactentes nascidos com peso insuficiente nos primeiros dois anos de vida [dissertação]. São Paulo: Faculdade de Medicina da Universidade de São Paulo; 1998. 
21. World Health Organization Expert Comitee. Physical status: the use and interpretation of anthropometry. Genebra; 1995. 452 p.

22. Silva EM, Miranda CT, Puccini RF, Nobrega FJ. Day care centres as an institution for health promotion among needy children: an analytical study in Sao Paulo, Brazil. Public Health. 2000; 114: 385-8.

23. Bueno MB, Marchioni DML, Fisberg RM. Evoluçäo nutricional de crianças atendidas em creches públicas no Município de Säo Paulo, Brasil. Rev Panam Salud Publica. 2003; 14: 165-70.

24. Taddei JA, Cannon MJ, Souza P, Vitalle S, Nóbrega FJ. Nutritional gains of underprivileged children attending a day care center in Säo Paulo City, Brazil: a nine month follow-up study. Rev Bras Epidemiol. 2000; 3: 29-37.

25. Marcondes E, Berquo ES, Luongo J, Yunes J, Martins SS, Hegg R. Estudos antropométricos de crianças brasileiras de zero a doze anos de idade. Anais Nestlé. 1970; 84: 1-200.

Recebido em 13 de dezembro de 2008

Versão final apresentada em 13 de agosto de 2009

Aprovado em 3 de setembro de 2009
26. Kirkwood BR. Essentials of medical statistics. London: Blackwell Sciences Ltd; 1988. 234 p.

27. Kay's SK, Hindmarsh PC. Catch-up growth: an overview. Pediatr Endocrinol Rev. 2006; 3: 365-78.

28. Pietiläinen KH, Kaprio J, Räsänen M, Rissanen A, Rose RJ. Genetic and environmental influences on the tracking of body size from birth to early adulthood. Obes Res. 2002; 10: 875-84.

29. Monset-Couchard M, de Bethmann O, Relier JP. Long term outcome of small versus appropriate size for gestational age co-twins/triplets. Arch Dis Child Fetal Neonatal Ed. 2004; 89: F310-4.

30. Lansky S, França E, Leal MC. Avoidable perinatal deaths in Belo Horizonte, Minas Gerais, Brazil, 1999. Cad Saúde Pública. 2002; 18: 1389-400. 